\title{
On the thin regular geometries of rank four for the Janko group $J_{1}$
}

\author{
Michael Ian Hartley Dimitri Leemans
}

\begin{abstract}
The Janko group $J_{1}$ acts regularly on six rank four thin residually connected geometries. Two of them are polytopes of type $\{5,3,5\}$ and $\{5,6,5\}$. In this paper, we show that starting from the $\{5,3,5\}$ polytope, the five other thin geometries may be constructed in a simple manner.
\end{abstract}

Keywords : abstract polytopes, thin geometries, sporadic group

MSC 2000: 51M20, 20F65, 52B15

\section{Introduction}

In [6], Dimitri Leemans used a series of MAGMA programs in order to classify for some groups all thin residually connected geometries on which these groups act regularly (see Section 2 for definitions). At that time, such a classification for the first Janko group was accessible but Leemans did not include it in the paper since the number of rank three geometries was too vast. In Figure 1, we give the diagrams of the six rank four geometries obtained (there are none of rank higher than four).

Geometries $\Gamma_{1}$ and $\Gamma_{2}$ have a linear diagram and therefore are abstract polytopes. The first one was discovered independently by Michael Hartley and led to the discovery of the universal locally projective polytope of type $\{5,3,5\}$ whose group of automorphisms is $J_{1} \times L_{2}(19)$ (see [5] for more details).

In this paper we give constructions of all six thin residually connected geometries mentioned above. We show that, starting from $\Gamma_{1}$, we may reconstruct the five other geometries. The reconstruction is done via two operations that are examples of the general "mixing" operations mentioned in Section 7A of McMullen and Schulte's book on polytopes [8]. In a mixing operation, a new polytope or geometry is constructed from another by selecting certain products 






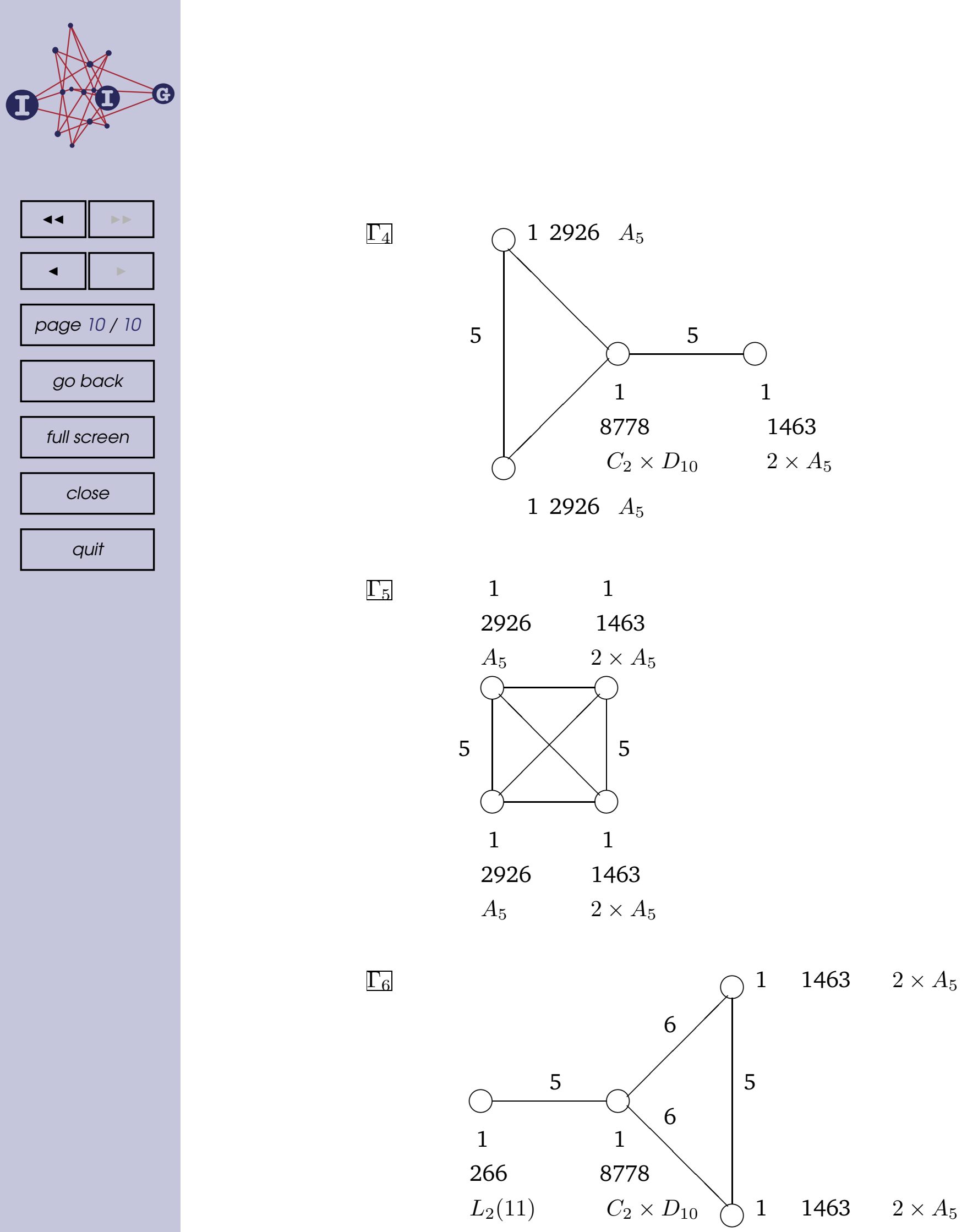

Figure 2: The rank four thin regular residually connected geometries of $J_{1}$ (II) 